\title{
Evaluation of Parotid Salivary Glucose Level for Clinical Diagnosis and Monitoring Type 2 Diabetes Mellitus Patients
}

\author{
Beibei Wang, ${ }^{1,2}$ Juan Du, ${ }^{1}$ Zhao Zhu, ${ }^{1}$ Zhihong Ma, ${ }^{1}$ Songlin Wang, ${ }^{1}$ and Zhaochen Shan ${ }^{1}$ \\ ${ }^{1}$ Oral and Maxillofacial Surgery Department, School of Stomatology, Capital Medical University, Beijing, China \\ ${ }^{2}$ Beijing Coloproctological Hospital (Beijing Erlonglu Hospital), Beijing, China \\ Correspondence should be addressed to Zhaochen Shan; shanzhch629@163.com
}

Received 27 August 2016; Accepted 16 January 2017; Published 31 January 2017

Academic Editor: Nicola Cirillo

Copyright (c) 2017 Beibei Wang et al. This is an open access article distributed under the Creative Commons Attribution License, which permits unrestricted use, distribution, and reproduction in any medium, provided the original work is properly cited.

\begin{abstract}
Background. To investigate the relationships among blood glucose, mixed saliva glucose, and parotid glucose in type 2 diabetes patients and to evaluate the diagnostic and monitoring value of salivary gland glucose in patients with type 2 diabetes (type 2DM). Material and Methods. Thirty patients with type 2DM and 30 healthy age- and sex-matched individuals were included in this study. Glucose levels in unstimulated mixed saliva and in unstimulated parotid saliva were measured by the glucose oxidase peroxidase method. Results. The blood glucose and parotid salivary glucose levels in type 2DM patients were significantly higher than those in the controls $(P<0.05)$. The blood glucose, parotid salivary glucose, and mixed salivary glucose were $7.46 \pm 1.44 \mathrm{mmol} / \mathrm{L}$, $0.18 \pm 0.19 \mathrm{mmol} / \mathrm{L}$, and $3.17 \times 10^{-2} \pm 2.84 \times 10^{-2} \mathrm{mmol} / \mathrm{L}$, respectively, in the type $2 \mathrm{DM}$ group; the corresponding glucose levels in the control group were $5.56 \pm 0.71 \mathrm{mmol} / \mathrm{L}, 7.70 \times 10^{-2} \pm 6.02 \times 10^{-2} \mathrm{mmol} / \mathrm{L}$, and $3.47 \times 10^{-2} \pm 2.79 \times 10^{-2} \mathrm{mmol} / \mathrm{L}$. The parotid salivary and blood glucose levels in type $2 \mathrm{DM}$ patients were strongly correlated; the linear regression equation for blood glucose and parotid salivary glucose was $Y=6.267 X+6.360$, with $r=0.810$. However, mixed salivary glucose levels were not significantly different in the type 2 diabetes group compared with the control group. Conclusion. Our results suggest that parotid salivary glucose has potential as a biomarker to monitor type 2DM and as a painless, noninvasive method for the management of type 2DM.
\end{abstract}

\section{Introduction}

Diabetes mellitus (DM) is a metabolic syndrome characterized by hyperglycemia and disturbances in the metabolism of carbohydrates, proteins, and lipids [1]. It is an endocrine disease characterized by a deficit in the production of insulin, which is responsible for lowering blood glucose concentration. Two etiologies of DM are recognized, type 1 and type $2[2,3]$. Type 1 diabetes is characterized by an absolute deficiency of insulin owing to the destruction of pancreatic beta cells, while type 2 is caused primarily by insulin resistance in peripheral target organs, such as the liver, muscle, and adipose tissue $[1,4]$.

The global prevalence of diabetes in the adult population is currently estimated at $6.4 \%$, and the number of people with diabetes is estimated to rise from 180 million in the year 2000 to 320 million in 2025 [5]. China, with 90 million people suffering from diabetes in 2010, has the largest diabetes population in the world, comprising $9.7 \%$ of the nation's adult population. Currently, diabetes is diagnosed by measuring blood glucose levels, and, because this generally requires a finger stick or venipuncture, it is physically and psychologically traumatic to the patients. Therefore, a simple, noninvasive, and painless procedure, such as measuring salivary glucose concentration, would be desirable.

Saliva is a biological fluid that can reflect local and systemic changes because the composition of saliva is influenced by the hormonal, immunologic, neurologic, nutritional, and metabolic state of the individual $[6,7]$. The incidence of caries, periodontal disease, and candidosis may be increased in individuals with elevated blood glucose levels $[8,9]$. Higher salivary glucose levels have been reported in diabetes patients compared with the levels in nondiabetics [9-12]. However, reports of the comparative values of blood and salivary glucose are inconsistent [13, 14]. These discrepancies may result from different population samples and use of different methods for collecting saliva or for glucose analysis. The poor correlation between blood and saliva glucose concentrations 
often seen in diabetes patients could result from oral retention of alimentary carbohydrates, glucose utilization by oral bacteria, release of carbohydrates from salivary glycoproteins, and contamination of saliva by a large outflow of crevicular fluid in patients with a poor gingival status [15]. However, saliva can be directly collected from the parotid glands using a Lashley cup [16] without being released into the oral cavity [17].

A previous study found that the glucose concentration in parotid secretions correlated differently with plasma glucose concentrations for each subject and at a much lower level compared with blood glucose. Although the mean glucose concentration in the parotid saliva of subjects with diabetes was slightly higher at each measurement compared with that of subjects without diabetes, there was considerable overlap of the concentration curves for individuals in each group. Therefore, further study of the correlation between parotid and plasma glucose concentrations is important because of the potential value in the diagnosis and monitoring of diabetes mellitus [18]. The present study was a comparative analysis of the glucose concentrations in mixed saliva from all salivary glands (i.e., saliva secreted into the oral cavity), unstimulated parotid glands, and blood glucose in patients with type 2DM and subjects without diabetes.

\section{Materials and Methods}

2.1. Subjects. The study was approved by Beijing Stomatologic Hospital ethics committee (number: 2014024), and all participants gave their informed consent. Thirty patients (13 male and 17 female) with type 2DM from 49 to 82 years of age (mean, $68.3 \pm 9.4$ years) and a control group of 30 clinically healthy subjects ( 15 male and 15 female) from 47 to 84 years of age (mean, $67.5 \pm 9.1$ years) were enrolled. All the participants were recruited at the Department of Geriatric Dentistry, Beijing Stomatologic Hospital, Capital Medical University between 2014 and 2016.

Individuals who smoked, suffered from alcoholism, were pregnant, were edentulous, had prior surgery of the salivary glands, and were being treated with radiotherapy of the head and neck region and those with Sjögren's syndrome, rheumatoid arthritis, or lupus erythematosus were excluded from this study. The control subjects were free of chronic diseases and did not take any medications other than vitamins or occasional analgesics.

2.2. Sample Collection. Resting saliva from the parotid gland and venous blood $(5 \mathrm{ml})$ were collected between 8:00 and 11:00 in the morning after an 8-hour fast; patients did not perform any oral hygiene in the $90 \mathrm{~min}$ before the saliva collection. The parotid duct orifice was visualized with a mouth mirror and rinsed with water. The parotid region was gently massaged until a small amount of clear fluid flew from the parotid gland duct. Then parotid saliva was collected using a Lashley cup in $10 \mathrm{~min}$ [16].

2.3. Mixed Saliva Collection. Subjects were asked to spit out or swallow their saliva and then stay still, passively allowing the saliva to drain over the lower lip into a preweighed test tube for 10 minutes.

2.4. Salivary Glucose Assay. The collected parotid saliva and mixed saliva were stored at $-80^{\circ} \mathrm{C}$ until use. The glucose oxidase peroxidase method (GOD-POD) was used to determine the salivary glucose level. Briefly, saliva samples were thawed and centrifuged at $3000 \mathrm{rpm}$ for 5 minutes, $4 \mu \mathrm{L}$ of the supernatant was transferred into Eppendorf tubes, and $2000 \mu \mathrm{L}$ of enzyme reagent from the glucose test kit (Leadman Biochemistry Co., Ltd, Beijing, China) was added to each sample. Three standards were also prepared. The salivary glucose assay mixtures were incubated in a water bath at $37^{\circ} \mathrm{C}$ for $5 \mathrm{~min}$ and were then transferred to $1.5 \mathrm{~mL}$ cuvettes. The absorbance was read at a wavelength of $505 \mathrm{~nm}$ with a spectrophotometer and the glucose level was determined by comparison to the standard solution.

Fasting blood glucose was tested before breakfast using a glucose meter (Roche Ltd, Switzerland). Briefly, the index finger was disinfected with $70 \%$ alcohol, and a disposable sterile needle was used to obtain a drop of blood, which was collected on a glucose test strip and then inserted into the glucose meter. The blood glucose level was determined and recorded on the patient's chart.

2.5. Statistics Analysis. All statistical calculations were performed using SPSS v.12 statistical software. Student's $t$-test was used to determine statistical significance, which was indicated by $P<0.05$ or $P<0.01$.

\section{Results}

3.1. Salivary Flow in DM Group and Control Group. Mixed salivary flow was $0.32 \pm 0.15 \mathrm{ml} / \mathrm{min}$ and $0.29 \pm 012 \mathrm{ml} / \mathrm{min}$ in patients with type 2 diabetes and the healthy subjects, respectively; the flow of mixed saliva was not significantly different in two groups. The level of the parotid salivary flow was not significantly different in patients $(0.05 \pm 0.03 \mathrm{ml} / \mathrm{min})$ with type 2 diabetes and control group $(0.04 \pm 0.03 \mathrm{ml} / \mathrm{min})$.

3.2. Glucose Levels in Blood and Saliva. To evaluate the diagnostic and monitoring value of single salivary gland glucose in patients with type 2 diabetes, we first determined the levels of blood, mixed salivary, and parotid salivary glucose in type 2 diabetes patients. The mean levels of blood, parotid salivary, and mixed salivary glucose were $7.46 \pm 1.44 \mathrm{mmol} / \mathrm{L}$, $0.18 \pm 0.19 \mathrm{mmol} / \mathrm{L}$, and $3.17 \times 10^{-2} \pm 2.84 \times 10^{-2} \mathrm{mmol} / \mathrm{L}$ in the type $2 \mathrm{DM}$ group and $5.56 \pm 0.71 \mathrm{mmol} / \mathrm{L}, 7.70 \times 10^{-2} \pm$ $6.02 \times 10^{-2} \mathrm{mmol} / \mathrm{L}$, and $3.47 \times 10^{-2} \pm 2.79 \times 10^{-2} \mathrm{mmol} / \mathrm{L}$ in the healthy subjects, respectively (Table 1). The blood glucose and parotid salivary glucose levels were significantly higher in the type 2DM group than in the control group. However, the mixed salivary glucose levels in the type 2DM and control groups were not significantly different (Table 1). Interestingly, the parotid salivary glucose level was significantly higher than the mixed salivary glucose level in both the type 2DM and control groups (Table 1). These results support the potential 
TABLE 1: The blood glucose, parotid salivary, and mixed salivary glucose results in DM and control groups.

\begin{tabular}{lccc}
\hline & $\begin{array}{c}\text { DM group } \\
(n=30)\end{array}$ & $\begin{array}{c}\text { Control group } \\
(n=30)\end{array}$ & $p$ \\
\hline $\begin{array}{l}\text { Blood glucose } \\
\text { Parotid salivary } \\
\text { glucose }\end{array}$ & $7.46 \pm 1.44$ & $5.56 \pm 0.71$ & 0.000 \\
$\begin{array}{l}\text { Mixed salivary } \\
\text { glucose }\end{array}$ & $3.17 \pm 2.84 \times 10^{-2}$ & $3.47 \pm 2.79 \times 10^{-2}$ & 0.681 \\
\hline
\end{tabular}

The units of glucose: $\mathrm{mmol} / \mathrm{L}$.

value of parotid saliva as a biomarker for monitoring diabetes mellitus.

3.3. The Correlation of Blood Glucose and Parotid Salivary Glucose. To assess the correlation of parotid salivary glucose with blood glucose levels in people with diabetes, we performed a regression analysis. As shown in Figure 1, the linear regression equation for parotid salivary glucose and blood glucose was $Y=6.267 X+6.360, r=0.810, p=0.000$. The results of linear regression indicated a significant relationship between the levels of parotid salivary glucose and blood glucose in the diabetes patients.

\section{Discussion}

Saliva, like plasma or serum, is a unique, complex body fluid, and the adequate production of saliva is essential for maintaining oral health. Normally, about $1000-1500 \mathrm{ml}$ of saliva is produced daily. Saliva is more than $99 \%$ water and contains sodium, potassium, glycoproteins, glucose, amino acids, and a variety of other substances. Previous studies have described the usefulness of saliva for the diagnosis of oral or systemic diseases such as periodontal diseases, oral squamous cell carcinoma, and human immunodeficiency virus (HIV) infection [19-21]. Advances in diagnostic technologies hold tremendous promise for achieving the long-term goal of developing clinically validated, saliva-based tests for health surveillance and early detection of oral disease and other systemic conditions.

In the present study, we found that the glucose levels in parotid saliva glucose were higher than in mixed saliva in both diabetes patients and controls. The blood glucose and parotid salivary glucose levels in type 2 diabetes patients were significantly higher than levels in the control group, and the glucose levels in parotid saliva were strongly correlated with the blood glucose levels in type 2 diabetes patients. However, there were no significant differences in the glucose levels in mixed saliva from diabetes patients and healthy controls after an 8-hour fast. These findings differ to some extent from those of other studies [22,23]. A previous study by Darwazeh et al. showed that, in diabetes patients, salivary glucose concentration was significantly higher than in controls and was directly related to blood glucose concentration [22]. These discrepancies may be related to the condition of the subjects included in the study. In our study, we included type 2DM patients with routine medication control, which may have

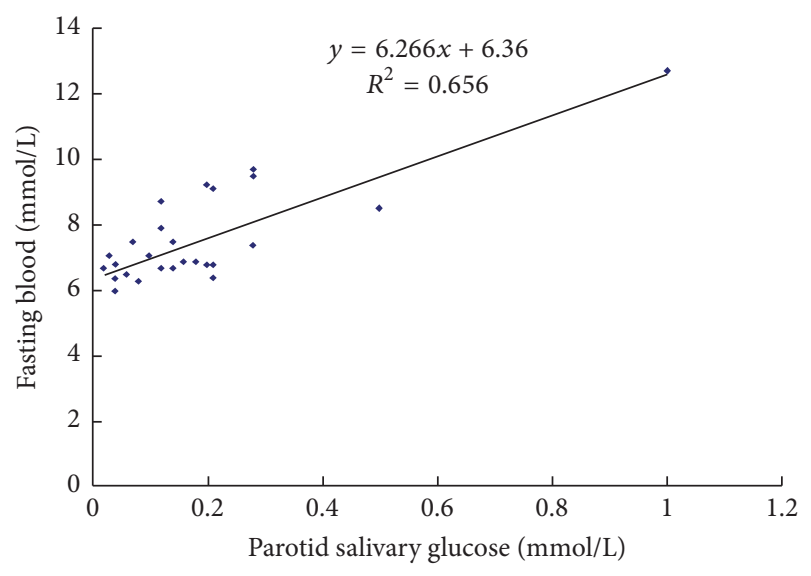

FIGURE 1: The correlation between parotid salivary glucose and blood glucose in DM group, correlation coefficient $r=0.810$.

resulted in the low blood and salivary glucose concentrations observed. In another study, no correlation was found between saliva and plasma glucose levels [10]. Nonetheless, our study revealed a significant, strong correlation between parotid salivary and blood glucose levels but not between mixed salivary and blood glucose levels; the glucose level in parotid saliva but not that in mixed saliva may thus reflect the blood glucose level.

The advantages of salivary assessment include noninvasive collection and cost effectiveness for screening of large populations [17]. Saliva is currently recognized as an excellent diagnostic biomarker of human body characteristics. The saliva microbiome, for example, is reputed to be biometrically as accurate as a fingerprint when used for screening characteristics for normality [21]. There is a possibility that saliva could be used as an alternative for blood in some laboratory tests, for example, to detect viral infections, such as HIV and $\mathrm{HCV}$, as a noninvasive procedure and allowing multiple sampling [24, 25].

Either single or mixed saliva can be collected, and it should be noted that many unknown factors and unstable elements influence the properties of mixed saliva. Saliva collected directly from a single gland is stable and not influenced by oral conditions; thus it might accurately reflect the status of blood glucose. Parotid saliva is easy to collect by both unstimulated and stimulated conditions (by chewing or acids). Dhanya and Hegde [9] reported that the salivary glucose concentration was higher when saliva was collected under unstimulated than under stimulated conditions. Other studies found no significant differences in glucose concentrations in saliva collected under unstimulated and stimulated conditions $[13,14]$. Since patients may not readily accept chewing or acid stimulation, and the moisture content is higher in stimulated saliva; unstimulated saliva may be more representative of the normal physiological state. In this study, we collected parotid and mixed saliva from type $2 \mathrm{DM}$ patients and healthy subjects under unstimulated conditions.

In our study, we found the salivary glucose level was higher in the type 2DM group than in the control group. 
These results are in line with studies of the ultrastructure of rat salivary glands in an experimental diabetes model. The parotid gland cells had swollen intracytoplasmic mitochondria without visible cristae. Mitochondria are the primary structures of aerobic oxidation; thus, intracellular glucose aerobic oxidation was disrupted in this diabetes model [26]. Another study showed that the permeability of the parotid basal cell membrane was increased in diabetic patients, resulting in substantial glucose oversecretion from the parotid duct [27]. That may be the reason for the increased glucose concentration in parotid saliva of type 2DM patients. However, there are still many issues that need to be addressed and further explored. For example, how to improve the sensitivity for glucose detection due to its low level in saliva as compared to serum and to standardize sample collection methods prior to analysis. Therefore, it is important to develop more sensitive methods for detecting salivary glucose that are suitable for convenient self-management by DM patients.

\section{Conclusion}

Our results demonstrated that parotid salivary glucose has potential as an indicator to monitor type $2 \mathrm{DM}$ and a noninvasive method in the management of diabetic patients.

\section{Competing Interests}

The authors declare that they have no competing interests.

\section{Acknowledgments}

This study was supported by the National Natural Science Foundation of China (81070843 to Z. C. Shan) and National Key Research and Development Program of China (Grant no. 2016YFC1102604).

\section{References}

[1] P. Bakianian Vaziri, M. Vahedi, H. Mortazavi, Sh. Abdollahzadeh, and M. Hajilooi, "Evaluation of salivary glucose, IgA and flow rate in diabetic patients: a case-control study," Journal of Dentistry (Tehran, Iran), vol. 7, no. 1, pp. 13-18, 2010.

[2] M. Motta, E. Bennati, L. Ferlito, M. Passamonte, and M. Malaguarnera, "Value and significance of new diagnostic criteria of diabetes mellitus in older people," Archives of Gerontology and Geriatrics, vol. 45, no. 1, pp. 103-108, 2007.

[3] M. S. Soares, M. M. Batista-Filho, M. J. Pimentel, I. A. Passos, and E. Chimenos-kustner, "Determination of salivary glucose in healthy adults," Medicina Oral, Patologia Oral y Cirugia Bucal, vol. 14, no. 10, pp. e510-e513, 2009.

[4] E. M. Chavez, G. W. Taylor, L. N. Borrell, and J. A. Ship, "Salivary function and glycemic control in older persons with diabetes," Oral Surgery, Oral Medicine, Oral Pathology, Oral Radiology, and Endodontics, vol. 89, no. 3, pp. 305-311, 2000.

[5] H. Schneider, J. Shaw, and P. Zimmet, "Guidelines for the detection of diabetes mellitus-diagnostic criteria and rationale for screening," The Clinical Biochemist Reviews, vol. 24, no. 3, pp. 77-80, 2003.
[6] G. W. Carlson, "The salivary glands: embryology, anatomy, and surgical applications," Surgical Clinics of North America, vol. 80, no. 1, pp. 261-273, 2000.

[7] A. Wolff, L. Zuk-Paz, and I. Kaplan, "Major salivary gland output differs between users and non-users of specific medication categories," Gerodontology, vol. 25, no. 4, pp. 210-216, 2008.

[8] R. Ravindran, D. M. Gopinathan, and S. Sukumaran, "Estimation of salivary glucose and glycogen content in exfoliated buccal mucosal cells of patients with type II diabetes mellitus," Journal of Clinical and Diagnostic Research, vol. 9, no. 5, pp. ZC89-ZC93, 2015.

[9] M. Dhanya and S. Hegde, "Salivary glucose as a diagnostic tool in Type II diabetes mellitus: a case-control study," Nigerian Journal of Clinical Practice, vol. 19, no. 4, pp. 486-490, 2016.

[10] S. Gupta, S. V. Sandhu, H. Bansal, and D. Sharma, "Comparison of salivary and serum glucose levels in diabetic patients," Journal of Diabetes Science and Technology, vol. 9, no. 1, pp. 9-16, 2015.

[11] B. N. Satish, P. Srikala, B. Maharudrappa, S. M. Awanti, P. Kumar, and D. Hrgar, "Saliva: a tool in assessing glucose levels in diabetes mellitus," Journal of International Oral Health, vol. 6, no. 2, pp. 114-117, 2014.

[12] P. V. S. Lakshmi, E. Sridevi, A. J. Sai Sankar, M. G. Manoj Kumar, M. Sridhar, and B. Sujatha, "Diagnostic perspective of saliva in insulin dependent diabetes mellitus children: an in vivo study," Contemporary Clinical Dentistry, vol. 6, no. 4, pp. 443-447, 2015.

[13] C. Carda, N. Mosquera-Lloreda, L. Salom, M. E. Gomez de Ferraris, and A. Peydro, "Structural and functional salivary disorders in type 2 diabetic patients," Medicina Oral, Patología Oral y Cirugía Bucal, vol. 11, no. 4, pp. E309-E314, 2006.

[14] H. Ben-Aryeh, M. Cohen, Y. Kanter, R. Szargel, and D. Laufer, "Salivary composition in diabetic patients," Journal of Diabetic Complications, vol. 2, no. 2, pp. 96-99, 1988.

[15] J. C. Hase, D. Birkhed, F. Lagerlöf, and E. Thornqvist, "Oral retention of glucose at pharmacologically reduced salivary flow in man," Scandinavian Journal of Dental Research, vol. 102, no. 3, pp. 180-185, 1994.

[16] G. M. Whitford, J. E. Thomas, and S. M. Adair, "Fluoride in whole saliva, parotid ductal saliva and plasma in children," Archives of Oral Biology, vol. 44, no. 10, pp. 785-788, 1999.

[17] A. Borg and D. Birkhed, "Secretion of glucose in human parotid saliva after carbohydrate intake," Scandinavian Journal of Dental Research, vol. 96, no. 6, pp. 551-556, 1988.

[18] E. Kaufman and I. B. Lamster, "The diagnostic applications of saliva-a review," Critical Reviews in Oral Biology and Medicine, vol. 13, no. 2, pp. 197-212, 2002.

[19] C. S. Miller, J. D. Foley, A. L. Bailey et al., "Current developments in salivary diagnostics," Biomarkers in Medicine, vol. 4, no. 1, pp. 171-189, 2010.

[20] M. D. Williams, "Integration of biomarkers including molecular targeted therapies in head and neck cancer," Head and Neck Pathology, vol. 4, no. 1, pp. 62-69, 2010.

[21] M. R. Parisi, L. Soldini, G. D. Perri, S. Tiberi, A. Lazzarin, and F. B. Lillo, "Offer of rapid testing and alternative biological samples as practical tools to implement HIV screening programs," New Microbiologica, vol. 32, no. 4, pp. 391-396, 2009.

[22] A. M. G. Darwazeh, T. W. MacFarlane, A. McCuish, and P. J. Lamey, "Mixed salivary glucose levels and candidal carriage in patients with diabetes mellitus," Journal of Oral Pathology \& Medicine, vol. 20, no. 6, pp. 280-283, 1991.

[23] C.-O. Reuterving, "Pilocarpine-stimulated salivary flow rate and salivary glucose concentration in alloxan diabetic rats. 
Influence of severity and duration of diabetes," Acta Physiologica Scandinavica, vol. 126, no. 4, pp. 511-515, 1986.

[24] S. Aydin, "A comparison of ghrelin, glucose, alpha-amylase and protein levels in saliva from diabetics," Journal of Biochemistry and Molecular Biology, vol. 40, no. 1, pp. 29-35, 2007.

[25] A. D. Mata, D. Marques, S. Rocha et al., "Effects of diabetes mellitus on salivary secretion and its composition in the human," Molecular and Cellular Biochemistry, vol. 261, no. 1-2, pp. 137-142, 2004.

[26] W. S. Li, J. H. Zhu, and J. Y. Zhang, "The ultrastructure study on the salivary glands in experimental diabetes rats," Chinese Journal of Practical Stomatology, vol. 3, no. 2, pp. 116-117, 2001.

[27] V. A. Murrah, J. T. Crosson, and J. J. Sauk, "Parotid gland basement membrane variation in diabetes mellitus," Journal of Oral Pathology, vol. 14, no. 3, pp. 236-246, 1985. 


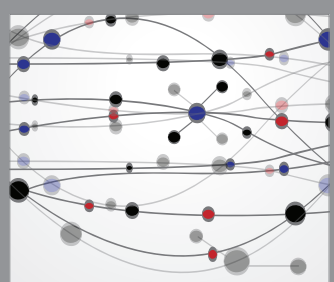

The Scientific World Journal
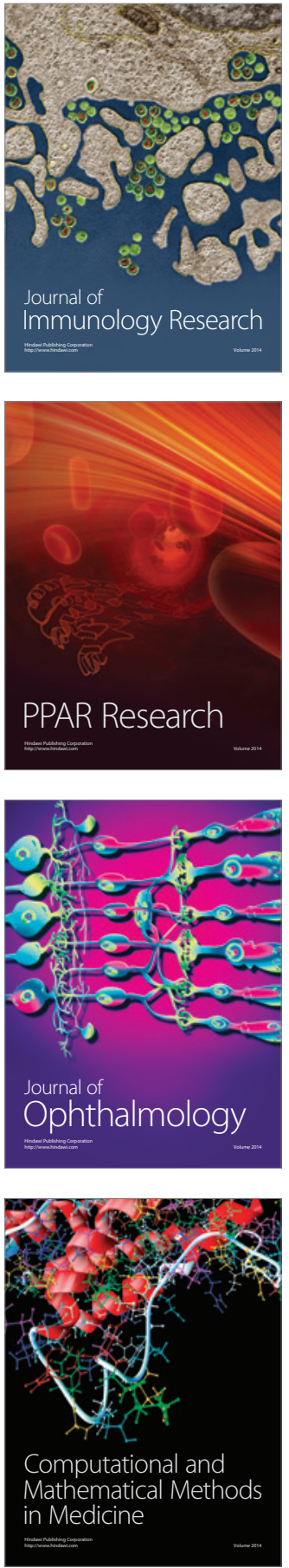

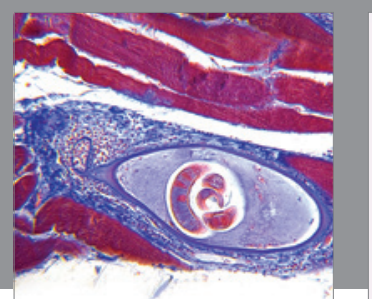

Gastroenterology Research and Practice
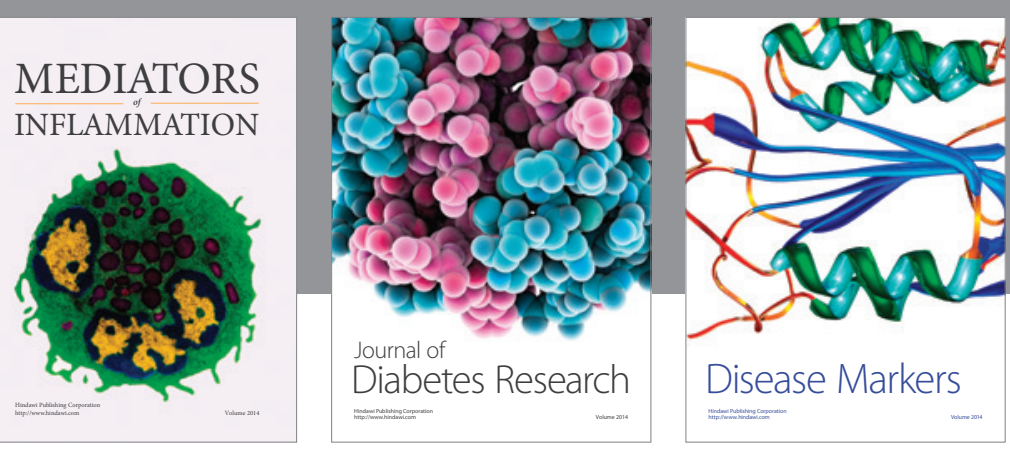

Disease Markers

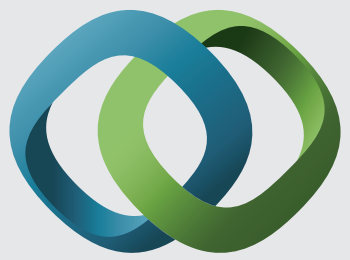

\section{Hindawi}

Submit your manuscripts at

https://www.hindawi.com
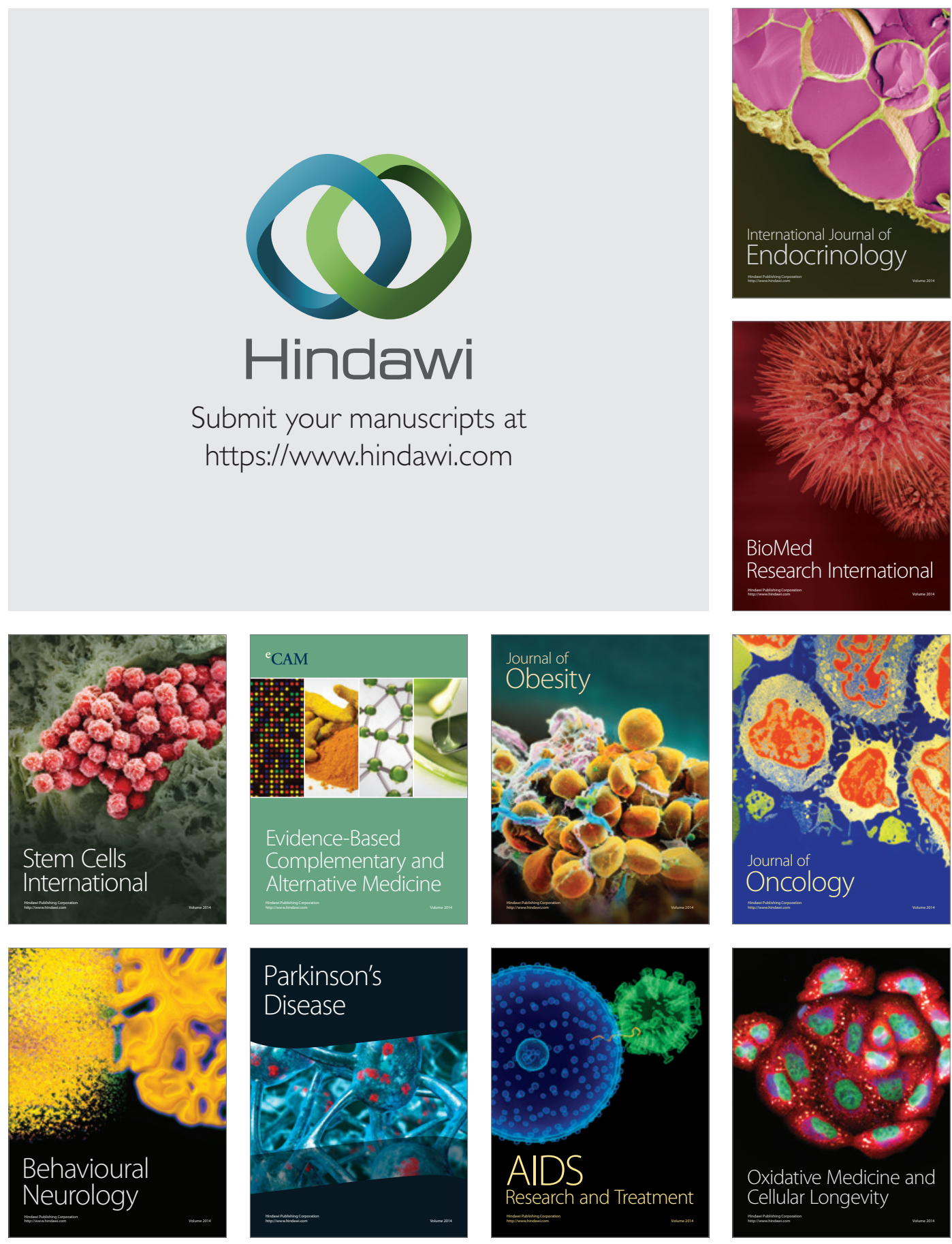